University of New Hampshire

University of New Hampshire Scholars' Repository

Space Science Center

Institute for the Study of Earth, Oceans, and

Space (EOS)

9-7-2012

\title{
Development of a telescope for medium-energy gamma-ray astronomy
}

\author{
Stanley D. Hunter \\ NASA Goddard Space Flight Ctr \\ Peter F. Bloser \\ University of New Hampshire, Peter.Bloser@unh.edu \\ Michael P. Dion \\ NASA Goddard Space Flight Ctr \\ Georgia A. DeNolfo \\ NASA Goddard Space Flight Ctr \\ Jason S. Legere \\ University of New Hampshire, jslegere@unh.edu
}

See next page for additional authors

Follow this and additional works at: https://scholars.unh.edu/ssc

Part of the Astrophysics and Astronomy Commons

\section{Recommended Citation \\ Stanley D. Hunter ; Peter F. Bloser ; Michael P. Dion ; Georgia A. DeNolfo ; Jason Legere ; Mark L. McConnell ; Suzanne F. Nowicki ; James M. Ryan ; Seunghee Son and Floyd W. Stecker " Development of a telescope for medium-energy gamma-ray astronomy ", Proc. SPIE 8443, Space Telescopes and Instrumentation 2012: Ultraviolet to Gamma Ray, 84430F (September 7, 2012); doi:10.1117/12.925216; http://dx.doi.org/10.1117/12.925216}

This Conference Proceeding is brought to you for free and open access by the Institute for the Study of Earth, Oceans, and Space (EOS) at University of New Hampshire Scholars' Repository. It has been accepted for inclusion in Space Science Center by an authorized administrator of University of New Hampshire Scholars' Repository. For more information, please contact Scholarly.Communication@unh.edu. 


\section{Authors}

Stanley D. Hunter, Peter F. Bloser, Michael P. Dion, Georgia A. DeNolfo, Jason S. Legere, Mark L. McConnell, Suzanne F. Nowicki, James M. Ryan, Seunghee Son, and Floyd Stecker 


\title{
Development of a telescope for medium-energy gamma-ray astronomy
}

Stanley D. Hunter ${ }^{{ }^{a}}$, Peter F. Bloser ${ }^{b}$, Michael P. Dion ${ }^{\mathrm{a}}$, Georga A. DeNolfo, Jason Legere ${ }^{\mathrm{b}}$, Mark L. McConnell', Suzanne F. Nowicki ${ }^{\mathrm{a}}$, James M. Ryan ${ }^{\mathrm{b}}$, Seunghee Son ${ }^{\mathrm{a}}$, and Floyd W. Stecker ${ }^{\mathrm{a}}$

${ }^{a}$ NASA/Goddard Space Flight Center, Greenbelt Road, Greenbelt, MD 20771;

${ }^{\mathrm{b}}$ University of New Hampshire, Department of Physics, Durham, NH, 03284

\begin{abstract}
The Advanced Energetic Pair Telescope (AdEPT) is being developed at GSFC as a future NASA MIDEX mission to explore the medium-energy (5-200 MeV) gamma-ray range. The enabling technology for AdEPT is the ThreeDimensional Track Imager (3-DTI), a gaseous time projection chamber. The high spatial resolution 3-D electron tracking of 3-DTI enables AdEPT to achieve high angular resolution gamma-ray imaging via pair production and triplet production (pair production on electrons) in the medium-energy range. The low density and high spatial resolution of 3DTI allows the electron positron track directions to be measured before they are dominated by Coulomb scattering. Further, the significant reduction of Coulomb scattering allows AdEPT to be the first medium-energy gamma-ray telescope to have high gamma-ray polarization sensitivity.

We review the science goals that can be addressed with a medium-energy pair telescope, how these goals drive the telescope design, and the realization of this design with AdEPT. The AdEPT telescope for a future MIDEX mission is envisioned as a $8 \mathrm{~m}^{3}$ active volume filled with argon at $2 \mathrm{~atm}$. The design and performance of the 3-DTI detectors for the AdEPT telescope are described as well as the outstanding instrument challenges that need to be met for the AdEPT mission.
\end{abstract}

Keywords: Gamma ray, telescope, pair production, polarimeter, time projection chamber

\section{INTRODUCTION}

Since the launch of AGILE and Fermi/LAT, the scientific progress in high-energy ( $E_{\gamma} \gtrsim 200 \mathrm{MeV}$ ) gamma-ray science has been extensive. Both of these telescopes cover a broad energy range from $\sim 20 \mathrm{MeV}$ to $>10 \mathrm{GeV}$. However, neither instrument is optimized for observations below $\sim 200 \mathrm{MeV}$ where many astrophysical objects exhibit unique, transitory behavior, such as spectral breaks, bursts, and flares. While Fermi and AGILE are expected to continue to make significant progress for the next several years, it is important to continue to develop instrumentation suitable for a follow-on gamma-ray mission

Despite the success of AGILE and Fermi, there remains a significant sensitivity gap in the medium-energy $(\sim 0.1-200$ $\mathrm{MeV}$ ) regime. The lower, and upper, portions of this range, below and above $\sim 30 \mathrm{MeV}$, have been explored only with COMPTEL and EGRET on CGRO. We believe the next major step in gamma-ray astrophysics must be a mediumenergy gamma-ray mission with significant improvements in angular resolution, and polarization sensitivity compared to current and previous instruments. We review some of the most significant medium-energy science goals below and describe the contributions that can be expected from a medium-energy telescope.

\subsection{Medium-Energy Gamma-Ray Astrophysics}

Since the formation of stars and galaxies shortly after the Big Bang, matter has undergone stellar formation and evolution ending in novae or supernovae that eject energetic particles and nuclei forming new generations of matter and stars. The goal of medium-energy gamma-ray astrophysics is to provide unique and direct insight into this cycle through the study of the accompanying gamma-ray emissions.

Medium-energy gamma-ray astronomy was recognized as early as the SAS-2 era (Fichtel 1976) ${ }^{1}$ to have unique potential to make important contributions to several long-standing problems central to modern physics, astrophysics, and

*stanley.d.hunter@nasa.gov; phone 1301 286-7280; fax 1301 286-0677; http://www.nasa.gov

Space Telescopes and Instrumentation 2012: Ultraviolet to Gamma Ray,

edited by Tadayuki Takahashi, Stephen S. Murray, Jan-Willem A. den Herder,

Proc. of SPIE Vol. 8443, 84430F - @ 2012 SPIE · CCC code: 0277-786/12/\$18 - doi: 10.1117/12.925216

Proc. of SPIE Vol. $844384430 \mathrm{~F}-1$ 
cosmology. Potential contributions include the detection of gamma rays from dark matter annihilation processes (Rudaz \& Stecker $1988^{2}, 1991^{3}$ ), the measurement of the extragalactic background radiation fields and the strength of cosmic magnetic fields, detailed information about the astrophysical processes that lead to the growth of black holes, information about the earliest epoch of star-formation (Stecker 2007), and the study of the most extreme accelerators in the Universe. We highlight below several of the important phenomenon that can be better understood through gammaray observations.

Pulsars: The energy range from 0.1 to $100 \mathrm{MeV}$ is a critical one for pulsar studies. Theoretical models predict a transition at $\sim 10 \mathrm{MeV}$ between two components of the pulsar spectrum, viz., a higher energy one produced by primary electrons and a lower energy one produced by cascade pair electrons and positrons (Harding, et al. 2008) ${ }^{5}$. These two components are predicted to have different spectral and polarization characteristics. Science goal: study the spectrum and polarization of pulsars in the medium-energy range.

Supernova Remnants: The turbulent nature of SNRs (Jokipii, 2007) ${ }^{6}$ suggests that small-scale structures, i.e. knots, are the locations for a majority of particle acceleration. New breakthroughs in Imaging Air Cherenkov Telescopes (IACT) have revealed photon signals from a number of potential sites of cosmic-ray acceleration, with spatially and spectrally resolved TeV images of a number of shell-type SNR and pulsar wind nebulae (Aharonian, et al. 2006) ${ }^{7}$. However, even with the excellent spectral and spatial resolution of these measurements, there is still no smoking-gun signature for hadronic processes ( $\pi^{0}$ production and decay) versus leptonic (inverse Compton) emission. Differentiation between these two processes can be made by a gamma-ray instrument with angular resolution several times that of Fermi for gamma rays with energies below $\sim 200 \mathrm{MeV}$ where the pion signature resides. Science goal: determine the primary emission process in SNRs, inferring the origin of Galactic cosmic rays.

Galactic Diffuse Emission: Mapping the Galactic diffuse emission on an angular scale of $<0.5^{\circ}$ (at $100 \mathrm{MeV}$ ) will allow individual SNRs, boundaries of super bubbles, and large-scale Galactic structure to be examined for evidence of the origins of cosmic ray acceleration and transport, mapping the transition from electron dominated to hadron dominated processes. EGRET, for example, was able to resolve the electron/hadron spectral transition (Hunter, et al., $1997)^{8}$. Science goal: determine the spatial transition and overall cosmic ray electron to proton ratio in the Galaxy.

Extragalactic Diffuse Gamma-Ray Emission: The extragalactic diffuse gamma-ray spectrum below $30 \mathrm{MeV}$ is not well understood (Sreekumar, Stecker, \& Kappadath, 19979; Stecker \& Salamon, 20010 ${ }^{10}$; Stecker \& Venters, $2011^{11}$ ). Observations in this range are important for searching for signals from new particles, such as WIMPs (Rudaz \& Stecker, $1991^{12}$ ) and gravitinos (Stecker, $1986^{13}$ ). The origin of the diffuse extragalactic gamma-ray spectrum below $200 \mathrm{keV}$, is now believed to be from radio-quiet AGN or Seyfert galaxies. Above $30 \mathrm{MeV}$, it is most likely from beamed radio-loud AGN, i.e. blazars. The gap below $\sim 30 \mathrm{MeV}$ may provide a window into other processes. Science goal: provide critical information in the MeV range to help determine the source of the extra-galactic background.

Gamma-Ray Blazars \& Other Active Galactic Nuclei: There is little information about gamma-ray AGNs below 100 $\mathrm{MeV}$. COMPTEL measurements of 3C273 indicated that some AGN can be most luminous from 10 to $30 \mathrm{MeV}$, below the Fermi-LAT energy range, a result confirmed by fits to Fermi-LAT Spectral Energy Distribution (Abdo et al. $2010^{14}$ ). Expanding our coverage of the AGN population in this little explored region will provide new insights into their frequency, spatial distribution, and variability. A promising test for synchrotron emission from blazars, e.g. 3C273 (Collmar, et al. $2000^{15}$ ), can be studied by measuring the gamma-ray polarization. Science goal: study AGN in a new critical energy range.

Testing Relativity with Polarization: Detection of polarization from extragalactic sources such as Gamma-ray Bursts (GRBs) and blazars has fundamental physics implications. In the general formulation of effective field theory for QED with Planck-scale Lorentz invariance violation, a vacuum birefringence effect will be produced caused by the resulting photon dispersion relation implying that photons of opposite helicity will have different phase velocities, an effect which increases with energy (Jacobson, et al. 2004 ${ }^{16}$; Mattingly, 2005 ${ }^{17}$; Stecker, $2011^{18}$ ). The difference in the photon rotation angles grows linearly with the distance to the source and with the square of the photon energy. This will destroy any intrinsic source polarization. As of now, polarization has only been probed at lower X-ray energies and will be further explored with the Gravity and Extreme Magnetism SMEX (GEMS, Swank et al. 2009 ${ }^{19}$ ). If gamma-ray polarization is definitely detected at $\mathrm{MeV}$ energies in cosmologically distance sources, this will place severe limits on Lorentz invariance violation. Such limits have implications for quantum gravity and string theories. Science goal: polarization studies provide an important probe for testing special relativity. 
Solar Flares: Above $10 \mathrm{MeV}$ solar flare emissions come from primary electron Bremsstrahlung and a mixture of secondary radiation of nuclear origin. Identifying and quantifying the separate components places strong constraints on acceleration and transport processes at the Sun. Furthermore, imaging the solar disk will help to distinguish between flare- and shock-related acceleration that may be manifested through distinct spatial footprints. . Science goal: observations of the solar disk at $\sim 100 \mathrm{MeV}$ would constrain models of particle acceleration.

\subsection{Medium-Energy Instrument Requirements and Design Drivers}

Achieving these science goals and filling the medium-energy sensitivity gap can only be fully realized with two gammaray telescopes optimized for gamma ray imaging via Compton scattering $(\sim 0.1-25 \mathrm{MeV})$ and pair production $(\sim 5-200$ $\mathrm{MeV}$ ), both with significant improvements in angular resolution and polarization sensitivity. McConnell \& Ryan ${ }^{20}$ (2004) review current and planned instruments below $30 \mathrm{MeV}$ capable of polarimetry measurements. In this paper we concentrate on the design of the Advanced Energetic Pair Telescope (AdEPT), a pair telescope optimized for imaging and polarization measurement of 5 to $200 \mathrm{MeV}$ gamma rays.

The design of all pair production space telescopes to date, SAS- $2^{21}$ (Derdeyn, 1972), COS-B ${ }^{22}$ (Bignami, et al. 1975), EGRET $^{23}$ (Kanbach, et al. 1988), AGILE $^{24}$ (Tavani, et al. 2003), and Fermi-LAT ${ }^{25}$ (Michelson, 2003), have utilized an electron tracking hodoscope consisting of a stack of detectors interleaved with thin metal foils positioned above a calorimeter. SAS-2, COS-B and EGRET utilized two-dimensional gas spark chambers whereas AGILE and Fermi/LAT have taken advantage of silicon-strip detectors (SSD). The multiple layers of metal foils provide substantial material for high interaction probability and large effective area. The conversion foils, however, also contribute to Coulomb scattering of the pair electrons which degrades the angular resolution (e.g. Hartman, $1989^{26}$ ) and polarization sensitivity (Mattox, $1990^{27}, 1991^{28}$ ). The hodoscope-calorimeter stack-up also tends to reduce the field of view (FOV) of the telescope, although the design of Fermi-LAT achieved a $\sim 2.5 \mathrm{sr}$ FOV by minimizing the height of the hodoscope.

A new approach to the design of a future pair telescope is required. This design must be optimized to achieve angular resolution approaching the pair production kinematic limit and the lowest possible minimum detectable polarization (MDP) by reducing the density of the conversion and scattering material per measurement interval of the hodoscope. The density per measurement interval, or granularity, can be reduced by decreasing the thickness of the conversion foils or increasing the separation between the measurement layers. For example, the Fermi-LAT front detector $(0.028 \mathrm{RL}$ tungsten foil plus $2 \times 0.004 \mathrm{RL}$ silicon strip detectors per $32.2 \mathrm{~mm}$ measurement layer, e.g. Bregeon, $2011^{29}$ ) has a granularity of $1.1 \times 10^{-3}$. If the tungsten foils were eliminated the granularity decreases to $2.5 \times 10^{-4}$. Zych et al. $\left(1999^{30}\right)$ proposed this modification to a single LAT tower to improve the LAT angular resolution below $100 \mathrm{MeV}$. The change would have slightly reduced the overall effective area of the LAT, but would have provided nearly equal sensitivity because of the improvement in angular resolution. Buehler et al. $\left(2010^{31}\right)$ estimated that Fermi LAT might have marginal polarization sensitivity by selecting $50-200 \mathrm{MeV}$ photons that convert in the silicon rather than the tungsten foils. Their analysis, omitting background and trial factor considerations, concluded that $20 \%$ polarization from Vela could be detected at $3 \sigma$ using 20 months of data.

These ideas to improve the angular resolution and polarization sensitivity of Fermi LAT are both based on reducing the granularity of the detector. Considering angular resolution and polarization sensitivity in terms of granularity provides a metric for the design of a gamma-ray telescope.

In the case of nuclear pair production, the accuracy with which the gamma-ray direction can be reconstructed from the electron, positron pair is limited by the unobserved nuclear recoil. To estimate the maximum allowable scattering thickness as a function of photon energy, we consider the mean (cross-section weighted) RMS deflection angle between the electron or positron and the photon (Sterns $1949^{32}$ ). This angle is plotted as curve (a) in Figure 1. The distance in radiation lengths (RL)

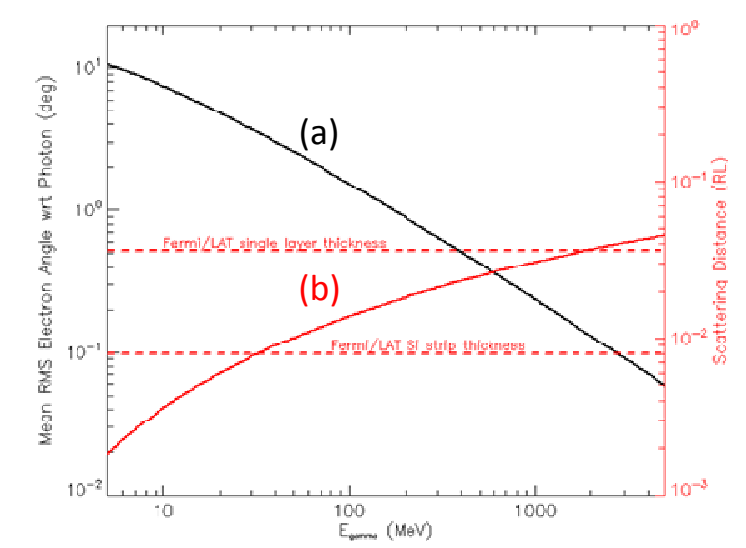

Figure 1 - (a) The mean, cross-section weighted, RMS deflection angle between the pair electron or positron and the incident gamma ray (Sterns, 1949). (b) The thickness of material, in radiation lengths, traversed by an electron with $\mathrm{E}_{\mathrm{e}}=\mathrm{E}_{\text {gamma }} / 2$ where the RMS scattering angle is equal to the RMS deflection angle. The thickness of one Fermi/LAT layer, with and without the tungsten foil is indicated by the dashed lines. 
through which an electron of mean energy $\left(\sim \mathrm{E}_{\gamma} / 2\right)$ travels before the RMS multiple Coulomb scattering equals the RMS deflection angle is plotted as curve (b) in Figure 1. The thickness of one Fermi/LAT layer, two SSD layers with and without the tungsten foil, is also shown in Figure 1. We refer to this energy as the scattering energy, $E_{\mathrm{s}}$, the photon energy below which the direction of the pair electron and positron is dominated by Coulomb scattering after traversing a single measurement layer. Since this is the thickness of a single measurement layer the determination of the electron and positron direction improves as the square-root of the number of measurements (proportional to the electron energy) is increased until Coulomb scattering dominates the measurement. The direction of the electron and positron from photons with $E_{\gamma}>E_{\mathrm{s}}$ is limited by the depth and measurement resolution of the hodoscope.

Polarization sensitivity is also affected by the conversion material. Kel'ner et al. $\left(1975^{33}\right)$ have calculated the polarization modulation factor, $\lambda$, as a function of converter thicknesses. These calculations assume that the electron and positron energies are greater than $1.5 \mathrm{MeV}$ and that the angle between them and the photon is less than $40^{\circ}$. At all energies, $\lambda$ decreases rapidly as the converter thickness increases above a few times $10^{-3}$ RL, Figure 2 .

\section{AdEPT MIDEX MISSION}

Maximizing the angular resolution and polarization sensitivity requires that the electron and positron directions be measured before they traverse more than a few times $10^{-3} \mathrm{RL}$ of material. This requirement can only be met with a low density medium corresponding to a granularity less than $\sim 10^{-4}$. Historically this low granularity has been achieved with a whole genre of detectors based on gas physics including cloud chambers, bubble chambers, and gas-wire detectors (spark and drift chambers, etc., see e.g. Rice-Evans $1974^{35}$ ).

\subsection{3-DTI Detector Technology}

The enabling technology for AdEPT is the Three-Dimensional Track Imager (3-DTI) detector. The 3-DTI technology extends the lowdensity concept to the extreme, eliminating the conversion foils altogether and using a gas as both the gamma-ray conversion and electron tracking medium. The 3-DTI technology couples the low density of a gas detector with high-resolution 3-D charge particle track imaging by combining a Time Projection Chamber (TPC) with 2-D Micro-Well Detector (MWD) readout and Negative-Ion (NI) drift to provide high resolution electron tracking.

The 2-D Micro-Well Detector (MWD) consists of two orthogonal layers of electrodes separated by an insulating substrate, Figure 3. The cathode and anode electrode strips are etched into the top and bottom layers respectively of a copper clad insulator using state-of-the-art flex circuit board technology. Holes etched in the top (cathode) electrodes are concentric with blind vias in the insulator that expose the anode and form the micro-wells. Our MWD design has $200 \mu \mathrm{m}$ diameter $x$ $200 \mu \mathrm{m}$ deep wells on $400 \mu \mathrm{m} \times 400 \mu \mathrm{m}$ center-to-center pitch.

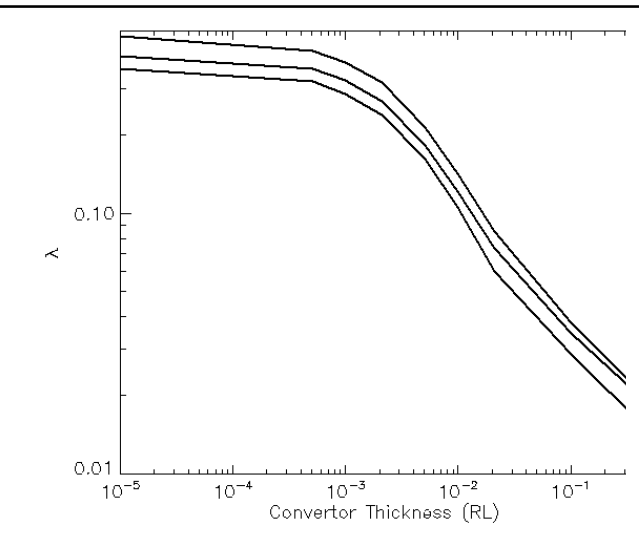

Figure 2 - Dependence of the modulation factor, $\lambda$, with thickness of material traversed. After Kotov $\left(1988^{34}\right)$.

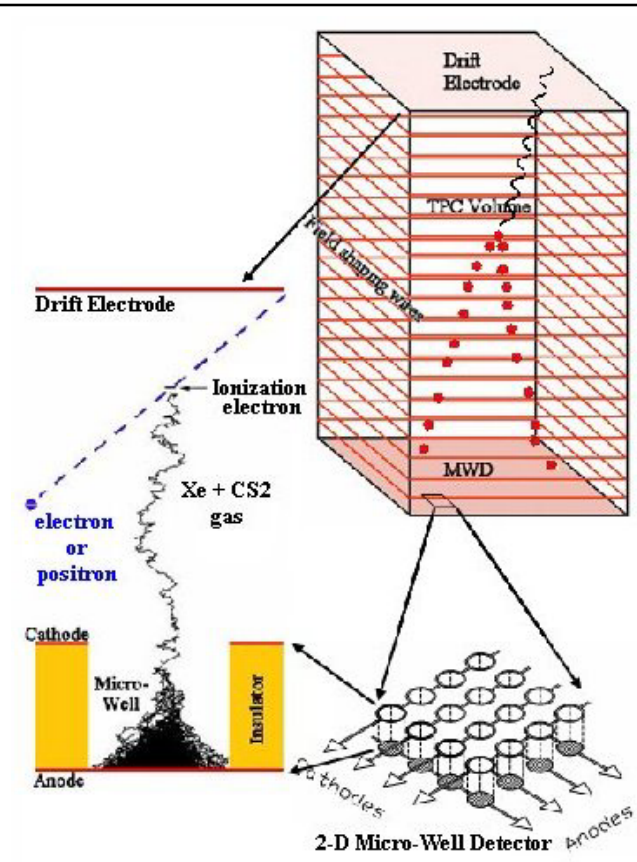

Figure 3 - Schematic of the 3-DTI showing the TPC volume, drift electrode and field shaping wires, and MWD. The e -pair from a gammaray conversion in the TPC traverses the gas leaving a trail of ionization charge that drifts toward the MWD array and into individual wells. Inset images show the anode and cathode structure of the MWD (lower right) and simulation of an avalanche in a micro-well (left).

The Time Projection Chamber (TPC) which defines the 3-DTI active volume is defined by a drift electrode on the top, a linear potential gradient field-shaping cage of wires, and a MWD array on the bottom. A charged particle produces 
primary ionization charge, proportional to $d E / d x$ left along its track. The charge is then amplified by Townsend avalanche (gas gain) by the MWD. The avalanche creates secondary electrons and positive ions that are collected by the anode and cathode electrodes of the MWD respectively. This provides 2-D spatial location X,Y of the ionization charge. The third dimension $\mathrm{Z}$ (height) is calculated from the uniform drift velocity of the ionization charge through the gas volume.

Operating the 3-DTI as a Negative Ion-Time Projection Chamber (NI-TPC) preserves primary track information over large distances due to the thermal limit diffusion of the NI carriers (Martoff, et al. 2000 $0^{36}$ ). This can be accomplished without the use of an applied magnetic field; contrary to drifting electrons. The drift velocity of free electrons in argon or xenon is $\sim 30 \mathrm{~mm} / \mu \mathrm{s}$ and depends strongly on additive gases $\left(\mathrm{CH}_{4}, \mathrm{CO}_{2}\right.$, etc.) and the drift field (Peisert \& Sauli, $\left.1984^{37}\right)$. In addition, the electron drift velocity is significantly higher than the thermal velocity of the gas, and the electrons have significant longitudinal and transverse diffusion that distorts the spatial structure of the ionization charge (track structure) after only a few $\mathrm{cm}$ of drift at moderate drift fields. The drift velocity of the ionization charge can be substantially reduced by the addition of a gas component with moderate electron affinity, e.g. $\mathrm{CS}_{2}$, to form negative ions. $\mathrm{CS}_{2}$ also provides a strong UV quencher, which reduces breakdown brought on by electron avalanche. Since thermal diffusion decreases with increasing electric field as $1 / \sqrt{E}$, the diffusion can be further reduced at the same time as increasing the drift velocity which reduces the occupancy, see below. Decreased diffusion preserves the spatial information of the primary charge even over long drift distances. Long drift distance allows for large active volume and thus increases the effective area of the 3-DTI that can be readout with one MWD. In the strong electric field of the micro-well, the ionization electrons are detached from the negative ions by collision (Dion, et al. $2010^{38}$ ) and then accelerated, producing a Townsend avalanche in the well.

\subsubsection{Diffusion measurement}

Negative ion drift is a relatively new concept and drift velocity data is not available in the literature. Therefore, a drift chamber was assembled to characterize the drift velocity and diffusion for 3-DTI gas mixtures. The measured drift velocity and diffusion results for $\mathrm{Xe}+\mathrm{CS}_{2}$ are shown in Figure 4. The drift velocity, and hence mobility $\left(\mathrm{V}_{\mathrm{d}} / \mathrm{E}\right)$ have also been measured with $<2 \%$ deviation for $\mathrm{CH}_{4}+\mathrm{CS}_{2}$ (Dion, et al. 2011 $1^{39}$ ). The longitudinal diffusion for negative ions after $50 \mathrm{~cm}$ drift distance at $1 \mathrm{kV} / \mathrm{cm}$ is $\sim 500 \mu \mathrm{m}$. The corresponding electron diffusion in $\mathrm{Ar}+\mathrm{CO}_{2}$ (see red line in Figure 4a) (Piuz $1983^{40}$ ) occurs only after $2.8 \mathrm{~cm}$ of drift.

\subsubsection{Electron tracks and Gamma-Ray Imaging}

The imaged track of an electron-positron pair from a 6.129 $\mathrm{MeV}$ gamma ray interacting in $80 \% \mathrm{P}-10+20 \% \mathrm{CS}_{2}$ at $0.55 \mathrm{~atm}$ is shown in Figure 5. This image was obtained

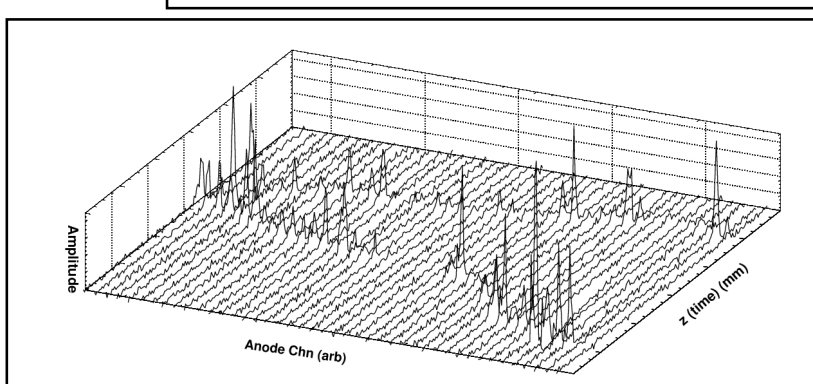

Figure 5 - A gamma-ray interaction from ${ }^{210} \mathrm{Pu}$ with ${ }^{13} \mathrm{C}$ target using the GEM amplified MWD detector. The gain of the GEM-MWD detector was $\sim 4000$. The amplitude is $d E / d x$ measured in the anode wells.

using a GEM amplified MWD detector. Several gamma-ray interactions were obtained using a $5 \times 5 \times 9 \mathrm{~cm}^{3} 3$-DTI prototype (de Nolfo $2008^{41}$ ). The derived angular resolution from 13 of these reconstructed tracks, not corrected for near-field parallax, was $\theta_{68}=\sim 18^{\circ}$ (Hunter et al. $2010^{42}$ ), which agrees well with the calculated curves for AdEPT, red $*$ in Figure 6.

\section{ADEPT}

Our concept for the AdEPT MIDEX telescope has an active volume of $8 \mathrm{~m}^{3}$ composed of a $2 \times 2 \times 2$ array of $1 \mathrm{~m}^{3} 3$-DTI NI-TPC modules filled with a $\operatorname{Ar}(95 \%)+\mathrm{CS}_{2}(5 \%)$ mixture at $2 \mathrm{~atm}$ pressure $\left(\sim 6.1 \times 10^{3} \mathrm{~cm} / \mathrm{RL}\right)$. The 3-D spatial 
resolution, $400 \mu \mathrm{m}$, is determined by the MWD pitch giving a 3-D RMS resolution, $\sigma_{x, y, z}$ of $400 \mu \mathrm{m} / \sqrt{12} \approx 115 \mu$. The $2 \mathrm{~m}$ depth of AdEPT corresponds to $\sim 0.033 \mathrm{RL}$ and the granularity, $400 \mu \mathrm{m} / 6.1 \times 10^{3} \mathrm{RL} / \mathrm{cm}$, is less than $10^{-5}$. The requirement to measure the electron directions in less than a few times $10^{-3} \mathrm{RL}$, to achieve the angular resolution and polarization sensitivity, is readily met by this low granularity. The predicted performance of this telescope is discussed below.

A detailed description of the 3-DTI/NITPC module design and performance is beyond the scope of this paper (see Hunter et al. in preparation), however, the two major enabling aspects of the 3-DTI/NITPC concept and their positive $(+)$ and negative (-) impacts on the AdEPT telescope design are briefly discussed below. Solutions to these negative aspects, see below, are currently being addressed as part of our ongoing NASA funded 3-DTI development.

- The low negative ion diffusion results in a large detector volume instrumented with one readout plane.

+ Each module will have $\sim 5000$ channels of front-end and readout electronics consisting of a charge sensitive amplifier, shaping amplifier, and 10 bit ADC. The power density of each AdEPT module is estimated to be $\sim 30 \mathrm{~W} / \mathrm{m}^{3}$.

- Techniques must be developed to tile smaller $\left(\sim 25 \times 25 \mathrm{~cm}^{2}\right)$ area MWDs together and electrically interconnect the orthogonal electrodes to form $1 \mathrm{~m}^{2}$ area MWDs.

Solution: Tiling MWDs and interconnecting the electrodes is a straight forward mechanical issue. An extension of the an-isotropic conductors currently being used to connect the MWDs to the front-end electronics is a likely approach.

- The slow negative ion drift velocity, $\sim 15 \mathrm{~m} / \mathrm{s}$, has several impacts

+ The shaping time of the front-end electronics is increased to $\sim 1 \mathrm{us}$ and digitization rate is reduced to $\sim 100 \mathrm{kHz}$.

+ Continuous readout eliminates instrument dead-time and complete readout of all charged particle tracks in volume.

- The maximum drift or integration time of a $1 \mathrm{~m}^{3}$ volume is $\sim 67 \mathrm{~ms}$. For typical space environment backgrounds, we expect $\sim 200$ charged particle tracks in this integration time from cosmic-rays in a $1 \mathrm{~m}^{3}$ volume at any moment. The long charge persistence in NITPC leads to an occupancy fraction of $\sim 2 \%$ samples/channel and a very high raw data rate, 5000 channels $\times 200 \mathrm{kHz} \times 36$ bits $/$ sample $\times 0.02$ samples $/$ channel $=7.2 \times 10^{8} \mathrm{bits} / \mathrm{s}$. This data rate must be processed in real time to separate the gamma-ray pair interactions from cosmic ray tracks and low-energy photon interactions.

Solution: The on-board processing needs of AdEPT can be readily met with giga-bit per second space computing systems currently under development at GSFC Code 584.

- Time correlation between fast scintillators is impractical. Thus, 1) an anti-coincidence system cannot be used to eliminate cosmic-ray events in the volume and 2) a calorimeter system cannot be used to determine the electron and positron energies.

Solution: Complete readout of all tracks and fast on-board processing reduces the gamma-ray selection to be accomplished with an image recognition algorithm. The 3-D track image of the electron and positrons can be used to determine their energies from a combination of track length, $d E / d x$ energy loss, and Coulomb scattering.

+ Elimination of the anti-coincidence and calorimeter greatly reduces the mass of the AdEPT telescope and allows for the viable design of an $8 \mathrm{~m}^{3}$ volume within the MIDEX budget.

\subsection{Photon Angular Resolution}

Reducing the scattering energy, Figure 1 , to $5 \mathrm{MeV}$ requires a measurement thickness of less than $\sim 2 \times 10^{-3} \mathrm{RL}$. Although the granularity of 3-DTI is very low, Coulomb scattering still limits the number of voxels used to determine the direction of the electron and positron. Over the AdEPT energy range the optimum number of samples increases from $\sim 10$ to 200 voxels and the RMS electron direction error is $\theta_{\mathrm{RMS}} \approx 1.3 \cdot\left(5 \mathrm{MeV} / E_{\gamma}\right)^{0.6} \mathrm{deg}$. The RMS photon direction error is the quadrature sum of the electron and positron direction errors, and the unobserved nuclear recoil. The estimated angular resolution of AdEPT is shown in Figure 6 for 2 gas pressures. The AdEPT angular resolution at $\sim 100 \mathrm{MeV}$ is expected to be similar to the Fermi/LAT front angular resolution $\sim 1 \mathrm{GeV}$. 


\subsection{AdEPT Prototype Calibration}

As part of this program, we plan to calibrate an AdEPT prototype at Duke University HIGS accelerator that offers $100 \%$ polarized gammarays from $\sim 15$ to $50 \mathrm{MeV}$. From this calibration we will determine the optimum electron energy determination algorithms, gamma-ray direction and energy, and the energy dependent polarization modulation factor.

\section{CONCLUSION}

With 3-DTI technology it is now possible to explore the m-e regime with high angular resolution and polarization sensitivity. The performance is encouraging and the few instrument challenges are readily tractable. The best approach to achieving the science goals in the medium-energy regime is a two-instrument observatory consisting of a Compton telescope optimized for imaging at lower energies and the AdEPT telescope that significantly improves the angular resolution and polarization sensitivity at higher energies. Furthermore, these two telescopes with naturally overlapping energy ranges provide spectral information and observations over a historically challenging energy range.

However, AdEPT alone will provide unique observations in the 5 to $200 \mathrm{MeV}$ energy range that address many of the critical science goals discussed in the introduction. We expect to fly a $50 \mathrm{~cm}$ AdEPT prototype in the 2016-18 time-frame in preparation for a future MIDEX opportunity.

\section{REFERENCES}

[1] Fichtel, C. E., Kniffen, D. A., Thompson, D. J., Bignami, G. F., and Cheung, C. Y., "Significance of Medium-Energy Gamma-Ray Astronomy to the Study of Cosmic Rays," ApJ, 208, 211-219 (1976).

[2] Rudaz, S., and Stecker, F. W., “,” ApJ, 325, 16 (1988).

[3] Rudaz, S., and Stecker, F. W., “,” ApJ, 368, 406 (1991).

[4] Stecker, F.W., [Stellar Photon and Blazar Archaeology with Gamma-rays] (2007).

[5] Harding, A.K., et al., “,” ApJ, 680, 1378 (2008).

[6] Jokipii, J.R., \& Giacalone, J., “,” ApJ, 660, 336 (2007).

[7] Aharonian, F., et al., "," Astronomy and Astrophysics, 457, 899 (2006).

[8] Hunter, S.D., et al., "Egret Observations of the Diffuse Gamma-Ray Emission From the Galactic Plane” ApJ, 481, 205 (1997).

[9] Sreekumar, P., Stecker, F.W., \& Kappadath, S.C., "Proceedings of the Fourth Compton Symposium, Williamsburg, VA," AIP Conf. Proc. 410, 344 (1997).

[10] Stecker, F.W., and Salamon, M.H., "The Extragalactic Gamma-Ray Background”, 432 (2001).

[11] Stecker, F.W., and Venters, T.M., "Components of the Extragalactic Gamma Ray Background," ApJ 736, 40 (2011)

[12] Rudaz, S., and Stecker, F. W., “,” ApJ, 368, 406 (1991).

[13] Stecker, F.W., et al., “,” Phys. Rev. Letts, 56, 2551 (1986).

[14] Abdo, A.A., et al., “,” ApJ 716, 30 (2010).

[15] Collmar, W., et al., "The Fifth Compton Symposium, Proceedings of the fifth Compton Symposium, held in Portsmouth, NH” Proc. American Institute of Physics (AIP), 510, 591 (2000).

[16] Jacobson, T., Liberati, S., Mattingly, D., \& Stecker, F. W., “, , Phys. Rev. Let., 93 (2004).

[17] Mattingly, D., [Living Reviews in Relativity], 8, 5 (2005).

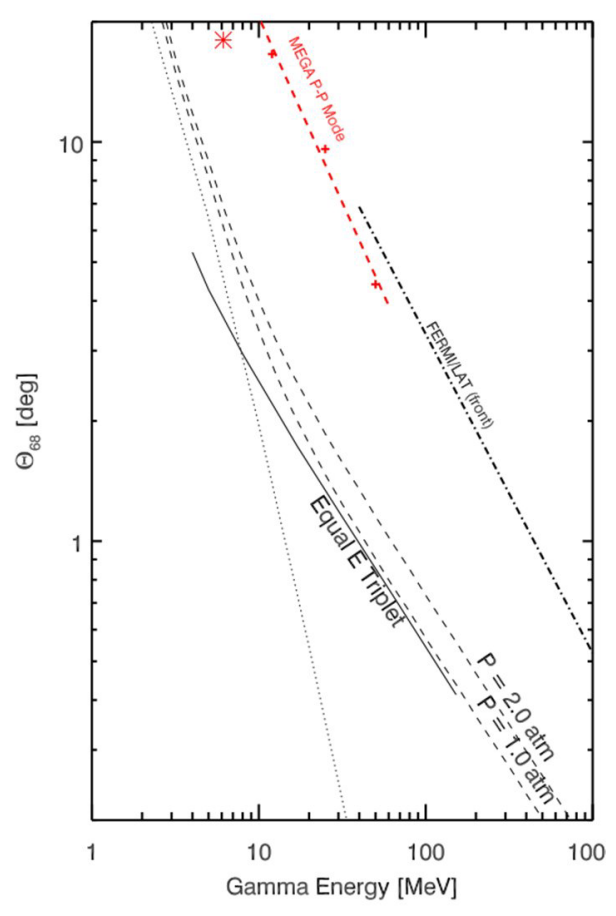

Figure 6 - The expected AdEPT angular resolution for nuclear pair production on Argon at 1 and 2 atm (dashed lines). The gamma-ray kinematic limit is determined by the unmeasured nuclear resolution (dash-dot line, FERMI/SSC) and the measured pair production angular resolution of the MEGA silicon-strip gamma-ray telescope (red points and line) are shown. 
[18] Stecker, F.W., "A new limit on the Plank scale Lorentz violation from gamma-ray burst polarization," Astropart. Phys. 35, 95-97 (2001).

[19] Swank, J., et al., "Gravity and Extreme Magnetism SMEX (GEMS)," X-ray Polarimetry: A New Window in Astrophysics, Rome, (2009).

[20] McConnell, M. L. \& Ryan, J. M., "Status and prospects for polarimetry in high energy astrophysics," New Astronomy Review, 48, 215 (2004).

[21] Derdeyn, S. M., “,” Nucl. Instr. and Meth. 98, 557 (1972).

[22] Bignami, G. F., et al., “,” Space Sci. Instrum. 1, 245 (1975).

[23] Kanbach, G., et al., “,” Space Sci. Rev. 49, 69 (1988).

[24] Tavani, M., et al., “,” Proc. SPIE 4851, 1151 (2003).

[25] Michelson, P. F., “,” Proc. SPIE 4851, 1144 (2003).

[26] Hartman, R. C., "Astronomical Gamma Ray Telescopes in the Pair Production Regime," Nucl. Phys. B, 10B, 130138 (1989).

[27] Mattox, J. R., Mayer-Hassewander, H. A., and Strong, A. W., “Analysis of the COS B Data for Evidence of Linear Polarization of Vela Pulsar Gamma Rays," ApJ, 363, 270-3 (1990).

[28] Mattox, J. R., "The Sensitivity of EGRET to Gamma-Ray Polarization,” Exp. Astro. 2, 75-84 (1991).

[29]Bregeon, J., "Design and performance of the silicon strip tracker of the Fermi Large Area Telescope," http://iopscience.iop.org/1748-0221/6/12/C12043.

[30]Zych, A. D., et al., "10-100 MeV Gamma-Ray Astronomy with GLAST," NASA Final Report, NRA 98-217-02 (1999).

[31]Buehler, R., et al., "Measuring polarization of gamma-rays with Fermi," SciNeGHE 2010 Trieste, http://scineghe2010.ts.infn.it/programmaScientifico.php

[32] Sterns, M., "Mean Square Angles of Bremsstrahlung and Pair Production," Phys. Rev. 76, 836-9 (1949).

[33] Kel'ner, S. R., Kotov, Yu. D., and Logunov, V. M., "Methods of measuring linear polarization of gamma quanta," Sov. J. Nucl. Phys. 21, 313-315 (1975).

[34] Kotov, Yu. D., “Methods of Measuring Gamma-Ray Polarization,” Space Sci. Rev., 49, 185-95, 1988

[35] Rice-Evans, P., [Spark, streamer, proportional and drift chambers], Richelieu Press, London (1974).

[36] Martoff, C. J., Snowden-Ifft, D. P., Ohnuki, T., Spooner, N., \& Lehner, M., “”” NIM, A440, 355 (2000).

[37] Peisert, A. and Sauli, F., "CERN Technical Report-84-08," CERN (1984).

[38] Dion, M.P., et al., "On the mechanism of Townsend avalanche for negative molecular ions," Astro. Phy., 33, 216220 (2010).

[39]Dion, M.P., et al., "Negative ion drift velocity and longitudinal diffusion in mixtures of carbon disulfide and methane," NIM, A648, 186-91 (2011).

[40] Piuz, F., "Measurements of the longitudinal diffusion of a single electron in gas mixtures used in proportional counters," NIM, 205, 425-36 (1983).

[41] de Nolfo, G.A., et al., "Gamma-Ray Imaging for Explosives Detection," SPIE, 6954 (2008).

[42] Hunter, S.D., et al., "Development of the Advanced Energetic Pair Telescope (AdEPT) for Medium-Energy Gamma-Ray Astronomy," SPIE Space Telescopes and Instrumentation, 7732:773221 (2010).

[43] Jost, R., Luttinger, J. M., \& Slotnick, M., "Distribution of Recoil Nucleus in Pair Production by Photons," Phys. Rev., 80, 189-96 (1950).

[44] G. Kanbach et al., "Development and calibration of the tracking Compton/Pair telescope MEGA," NIM A 541, 310$322(2005)$. 